\title{
Identification of Novel Ligands for the Z-DNA Binding Protein by Structure-Based Virtual Screening
}

\author{
Yang-Gyun Kim, ${ }^{a}$ Khac-Minh ThaI, ${ }^{b, 1)}$ Jieun Song, ${ }^{b}$ Kyeong Kyu KIm ${ }^{c}$ and Hyun-Ju PARK ${ }^{*}, b$ \\ ${ }^{a}$ Department of Chemistry, Sungkyunkwan University; ${ }^{b}$ College of Pharmacy, Sungkyunkwan University; Suwon, \\ Gyeonggi-do 440-746, Republic of Korea: and ${ }^{c}$ Department of Molecular Cell Biology, Samsung Biomedical Research \\ Institute, Sungkyunkwan University School of Medicine; Suwon, Gyeonggi-do 440-746, Republic of Korea. \\ Received September 19, 2006; accepted November 15, 2006
}

We describe the first discovery of small molecules that bind to the Z-DNA binding domain of human ADAR1 (Adenosine Deaminase Acting on RNA 1) by structure-based virtual screening of chemical database. These molecules bind to Z-DNA binding domain to inhibit the interaction with the Z-DNA. Many viruses have ZDNA binding proteins, which are structurally similar to Z-DNA binding domain of human ADAR1, and the ability of Z-DNA binding protein to bind the Z-DNA is essential for their pathogenicity. Therefore, the molecules identified in this study may serve as novel leads for the design of agents that inhibit biological functions of those pathogenic viruses.

Key words Z-DNA binding domain; Z-DNA; structure-based virtual screening

$\mathrm{Z} \alpha$, is a Z-DNA binding motif first identified from ADAR1 (Adenosine Deaminase Acting on double-stranded RNA 1), ${ }^{2,3)}$ and found in several proteins, e.g. vertebrate DLM-1 and viral E3L. An X-ray crystal structure of the Z $\alpha$ domain of human ADAR1 ( $\left.\mathrm{hZ} \alpha_{\mathrm{ADAR} 1}\right)$ has been solved in complex with Z-DNA. ${ }^{4,5)}$ Also, the NMR structure of the $Z \alpha$ domain of Vaccinia E3L revealed that the viral $Z \alpha$ domain has a topology similar to that described for the human $\mathrm{Z} \alpha$ domain. ${ }^{6}$

The role of Z-DNA binding in vaccinia virus pathogenesis has been addressed in recent studies. ${ }^{7,8)}$ The tenacity of the Z-DNA binding domain to bind Z-DNA is essential for the pathogenicity of many viruses in their hosts. Therefore, a ligand that disrupts the interaction between the Z-DNA binding domains and Z-DNA may inhibit biological functions of pathogenic viruses and lead to the development of a new class of antiviral agents.

In this contribution, we conducted a structure-based virtual screening of chemical database and biophysical analyses to discover small molecules that could bind to $\mathrm{hZ} \alpha_{\mathrm{ADAR} 1}$ and inhibit interaction with Z-DNA. The target receptor structure, an X-ray crystal structure of $\mathrm{hZ} \alpha_{\mathrm{ADAR} 1}$ bound to the lefthanded Z-DNA hexamer, d(CGCGCG $)_{2}$, reveals a detailed interaction of the protein to the DNA held together by 13 hydrogen bonds and 3 distinct van der Waals interactions (Fig. 1). ${ }^{4)}$ Seven amino acid residues, including $\operatorname{Arg} 174$ and Lys170, form hydrogen bonds primarily with the backbone phosphate groups of Z-DNA. The Tyr177 is involved as the only base contact seen in the complex that is a van der Waals contact with the exposed carbon 8 of the guanine base.

In the first step of virtual screening, a database search ${ }^{9,10)}$ was conducted to sort out ligands that match a three-dimensional pharmacophore criteria based on an X-ray structure. The pharmacophore query consists of a hydrophobic center on G4 and two hydrogen bond acceptor atoms (acceptor 1 and acceptor 2). Acceptor 1 is the anionic oxygen atom of G2-C3 phosphate which forms a water-mediated hydrogen bond with Asn173, and acceptor 2 is the anionic oxygen atom of C3-G4 phosphate which forms a hydrogen bond to the side-chain NH of Asn173. The distance between two hy- drogen bond acceptors is $5.85 \pm 0.2 \AA$ and the angle between acceptor 1-hydrophobic center-acceptor 2 is $50.69 \pm 5^{\circ}$. A total of 78425 entries from a commercially available chemical database ${ }^{11)}$ were searched, resulting in a reduction of the original dataset to 726 entries. In the second step, the 726 compounds were docked into the Z-DNA binding site of $\mathrm{hZ} \alpha_{\mathrm{ADAR} 1}$ using FlexX ${ }^{12)}$ and DOCK 4.0 program, ${ }^{13)}$ and ranked by FlexX scoring function and DOCK energy score, respectively. Our goal was to select compounds that ranked well by the two different docking algorithms and this yielded, 105 compounds which are ranked in the top 50 percent of both FlexX and DOCK outputs. As a final step of intensive docking analysis, the selected 105 compounds were docked into the active site using FlexX combined with the consensus scoring function, ${ }^{14)}$ Cscore. Visual inspection of the binding modes provided by FlexX, together with the score values from CScore were then used resulting in a focused set of 42 compounds for subsequent biophysical testing to examine the interaction with $\mathrm{hZ} \alpha_{\mathrm{ADAR} 1}$.

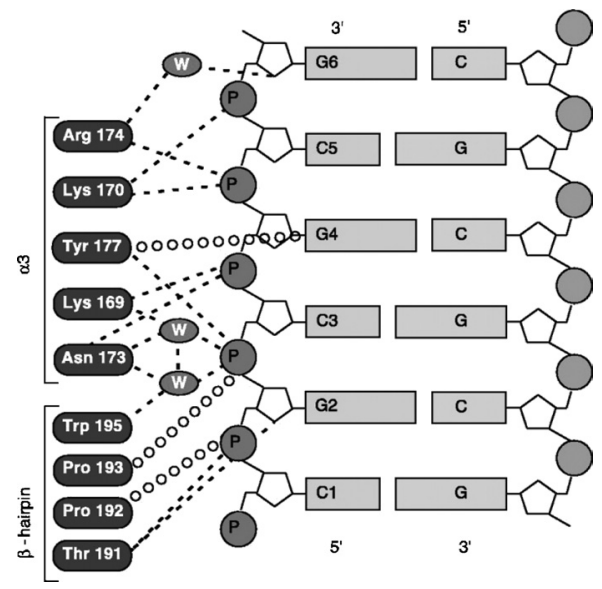

Fig. 1. Schematic Representation ${ }^{4)}$ of the Protein-Z-DNA Interactions Observed in X-Ray Crystal Structure of $Z \alpha_{\mathrm{ADAR} 1}$ : ds- $(\mathrm{CG})_{6}$ Complex (PDB id: 1QBJ)

Dashed lines represent H-bonds, open circles show van der Waals contacts, 'W' in oval indicates a water molecule in key positions within the protein-DNA interface. (Figure adapted from Schwartz et al., Science, 284, (1999) 1841-1845.) 
Surface plasma resonance (SPR) measurements ${ }^{15-17)}$ were conducted using a BIAcore 2000 system with the $\mathrm{hZ} \alpha_{\text {ADAR1 }}$ domain-immobilized streptavidin-coated sensor chips. The response unit values for tested compounds (at $1 \mathrm{~mm}$ in the flow solution) in the steady state region ranged from -20 to $220 \mathrm{RU}$, and among them, 10 compounds were shown to bind to $\mathrm{hZ} \alpha_{\mathrm{ADAR} 1}$ with significant $\mathrm{RU}$ values above 30 . For these 10 compounds, concentration dependency of the SPR response and the kinetics of binding to $h Z \alpha_{\mathrm{ADAR} 1}$ were determined by further BIAcore and simultaneous nonlinear regression analysis. Three compounds have shown a good correlation of sensorgram signals ${ }^{18}$ with their concentrations $(0.01-1 \mathrm{~mm})$, and the sensorgram for compound D2 is shown in Fig. 2, as an example. The hit compounds are noncharged small molecules containing several hydrogen bond acceptor atoms as illustrated in Fig. 3. It is notable that noncharged hit molecules are identified as a substitute for the ZDNA molecule, since virtual screening was conducted to target the binding pocket of $h Z \alpha_{\text {ADAR1 }}$ which primarily contacts with the negatively charged phosphate groups of Z-DNA.

To confirm the interaction of D2 with $h Z \alpha_{\mathrm{ADAR} 1}$ in the presence of Z-DNA, a gel migration shift assay was performed and $\mathrm{D} 2$ was shown to inhibit the binding of $\mathrm{hZ} \alpha_{\mathrm{ADAR} 1}$ to the Z-DNA oligomer duplex, $\mathrm{d}(\mathrm{CG})_{12}$, in a concentrationdependent manner (autoradiogram in Fig. 4). Complete inhi-

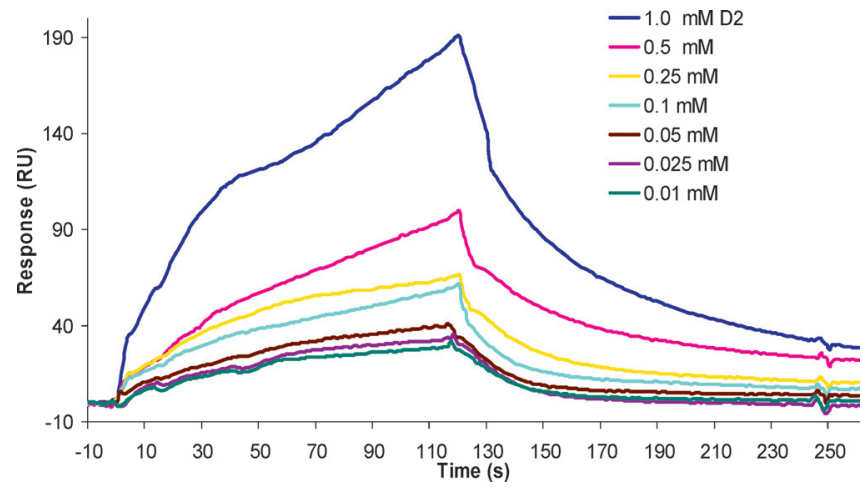

Fig. 2. SPR Sensorgram Showing the Binding of D2 to Immobilized $\mathrm{hZ} \alpha_{\mathrm{ADAR} 1}$ at Concentrantions Ranging from 0.01 to $1 \mathrm{~mm}(0.013,0.025$, $0.050,0.10,0.25,0.50,1.0 \mathrm{~mm}$ )

The sensorgrams were corrected by subtracting the signal of the reference cell. bition of Z-DNA binding was observed when about 10 -fold excess of D2 was added to the binding reaction, possibly due to the poor solubility of D2. It is predicted that a $1: 1$ interaction of $\mathrm{hZ} \alpha_{\mathrm{ADAR} 1}$ with Z-DNA would be inhibited by $\mathrm{D} 2$ at the concentrations much lower than the results shown in Fig. 4.

As shown in Figs. 5A and B, the FlexX-docked mode of $\mathrm{D} 2$ suggests that the compound interacts with $\mathrm{hZ} \alpha_{\mathrm{ADAR} 1}$ at

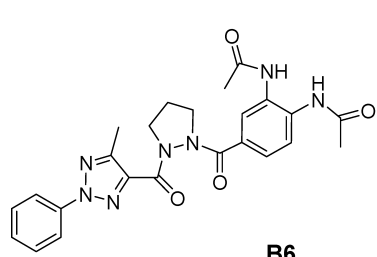

B6<smiles>CC(=O)Nc1ccc(N2CCCC2=O)c(NC(C)=O)c1</smiles>

D5<smiles>COc1ccc(Cl)cc1NC(=O)N1CCCN1C(=O)Cn1nnc(-c2ccccn2)n1</smiles>

Fig. 3. Structures of Hit Compounds That Have Been Shown to Bind to $\mathrm{hZ} \alpha_{\mathrm{ADAR} 1}$ with Concentration Dependency in BIAcore Assay

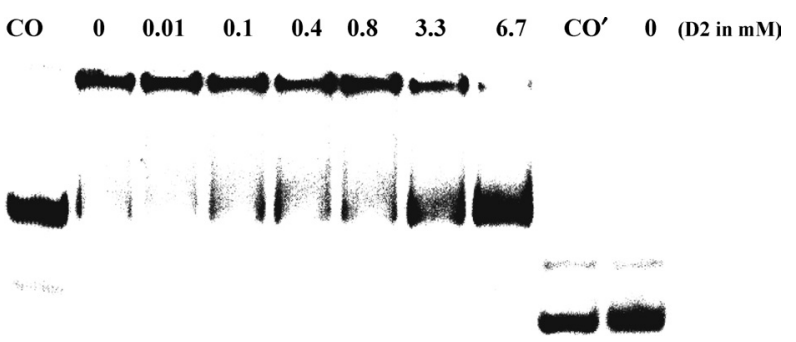

Fig. 4. Gel Mobility Shift Analysis of $h Z \alpha_{\mathrm{ADAR} 1}$ Binding to Z-DNA, in the Presence of D2

Lane $\mathrm{CO}$ and $\mathrm{CO}^{\prime}$ contain oligomer $\mathrm{d}(\mathrm{CG})_{12}$ and reference B-DNA $\mathrm{d}\left[\mathrm{CA}_{2} \mathrm{~T}_{3}(\mathrm{CG})_{2} \mathrm{~A}_{3} \mathrm{~T}_{2} \mathrm{G}\right]$, respectively. Other lanes are binding reaction mixtures of $\mathrm{hZ} \alpha_{\mathrm{ADAR} 1}(66.6 \mu \mathrm{M})$ and DNA duplex oligomers in the presence of D2 $(0-6.7 \mathrm{mM})$, after incubation in DNA binding buffer ( $15 \mathrm{~mm}$ Tris $\mathrm{pH} 7.9,80 \mathrm{~mm} \mathrm{KCl}, 4 \mathrm{~mm}$ DDT, 0.2 EDTA and $10 \%$ glycerol with $10 \mu \mathrm{g}$ of bovine serum albumin) at room temperature for $1 \mathrm{~h}$. The mixture was loaded onto native, low salt, $15 \%$ polyacrylamide gel and run in $0.5 \times \mathrm{TBE}$.

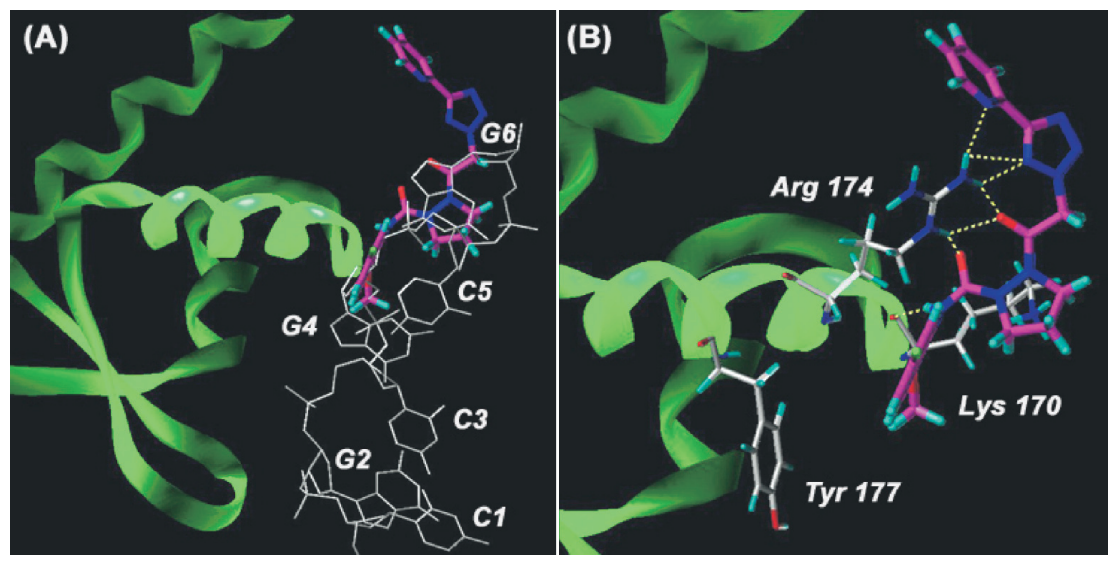

Fig. 5. (A) Docked Pose of D2 Overlapped over the X-Ray Pose of Z-DNA (Grey Line) Complexed with hZ $\alpha_{\text {ADAR1 }}$ (Green Ribbon).

(B) The Amino Acid Residues (Grey Carbon) Which Interact with D2 (Magenta Carbon) within the Binding Site Are Represented in Capped Stick

Colors of other atoms are red (oxygen), blue (nitrogen), and cyan (hydrogen). Hydrogen bonds between D2 and the amino acid residues (Arg174 and Lys170) are represented by yellow dotted lines $(<2.5 \AA)$ 
the site that is originally occupied by Z-DNA in the X-ray crystal structure (Fig. 5A). D2 interacts with key amino acids of $\mathrm{hZ} \alpha_{\mathrm{ADAR} 1}$ by forming multiple hydrogen bonds with Arg174 and Lys170, and a hydrophobic interaction with Tyr 177.

In this study, we first identified novel small molecules which bind to Z-DNA binding protein $\left(\mathrm{hZ} \alpha_{\mathrm{ADAR} 1}\right)$ to inhibit the interaction with Z-DNA by computational screening of chemical database, although further substantial optimization for binding affinity and solubility is necessary. The structures of these molecules would provide the basis for a second round of ligand-based virtual screening and design of the analogous ligands with increased binding affinity toward the Z-DNA binding proteins. The current virtual screening strategy can be applied to discover agents against viruses in which the Z-DNA binding protein is crucial for their pathogenicity. ${ }^{8,19)}$

Acknowledgments This research was supported by a grant (CBM2B513-001-1-0-0) from the Center for Biological Modulators of the 21 st Century Frontier R\&D Program, the Ministry of Science, Republic of Korea, and in part by the National Research Laboratory Program of the Korea Ministry of Science and Technology (NRL-2006).

\section{References and Notes}

1) Present address: Department of Medicinal Chemistry, Faculty of Life Sciences, University of Vienna, Vienna, Austria.

2) Herbert A., Alfken J., Kim Y.-G., Mian I. S., Nishikura K, Rich A., Proc. Natl. Acad. Sci. U.S.A., 94, 8421-8426 (1997).

3) Herbert A., Rich A., Proc. Natl. Acad. Sci. U.S.A., 98, 12132-12137
(2001).

4) Schwartz T., Rould M. A., Lowenhaupt K., Herbert A., Rich A., Science, 284, 1841-1845 (1999).

5) Ha S. C., Lowenhaupt K., Rich A., Kim Y.-G., Kim K. K., Nature (London), 437, 1183-1186 (2005).

6) Kahmann J. D., Wecking D. A., Putter V., Lowenhaupt K., Kim Y.-G., Schmieder P., Oschkinat H., Rich A., Schade M., Proc. Natl. Acad. Sci. U.S.A., 101, 2712-2717 (2004).

7) Kim Y.-G., Muralinath M., Brandt T., Pearcy M., Hauns K., Lowenhaupt K., Jacobs B. L., Rich A., Proc. Natl. Acad. Sci. U.S.A., 100, 6974-6979 (2003).

8) Kim Y.-G., Lowenhaupt K., Oh D.-B., Kim K. K., Rich A., Proc. Natl. Acad. Sci. U.S.A., 101, 1514-1518 (2004).

9) Martin Y. C., J. Med. Chem., 35, 2145-2154 (1992).

10) Hurst T. J., Chem. Inf. Comput. Sci., 34, 190-196 (1994).

11) LeadQuest Chemical Compounds Libraries (Version Feburary 2004), Tripos Receptor Research Ltd., U.K. 2004.

12) Rarey M., Kramer B., Lengauer T., Klebe G., J. Mol. Biol., 261, 470 489 (1996).

13) Ewing T. J. A., Makino S., Skillman A. G., Kuntz I. D. J., Comput. Aided Mol. Des., 15, 411-428 (2001).

14) Sybyl, 6.9 ed.; SYBYL molecular modeling software, Tripos Inc., St. Louis, MO, 2003.

15) Löpås S., Modern Drug Dis., 6, 47-49 (2003).

16) Myszka D. G., Rich R. L., Pharm. Sci. Tech. Today, 3, 310-317 (2000).

17) Fivash M., Towler E. M., Fisher R. J., Curr. Opin. Biotechnol., 9, 97101 (1998).

18) Markgren P.-O., Hämäläinen M., Danielson U. H., Anal. Biochem., 265, 340-350 (1998).

19) Ha S. C., Lokanath N. K., Van Quyen D., Wu C. A., Lowenhaupt K., Rich A., Kim Y. G., Kim K. K., Proc. Natl. Acad. Sci. U.S.A., 101, 14367-14372 (2004). 